\title{
AN INCIDENCE THEOREM FOR A CLASS OF CONTINUOUS REAL FUNCTIONS
}

\author{
V. J. BASTON AND F. A. BOSTOCK
}

\begin{abstract}
Sufficient conditions are obtained for a real function to have a line cutting its graph in exactly two points. Examples are given to show that none of these conditions may be omitted.
\end{abstract}

1. For some time an interest has been shown in subsets of $R^{2}$ having certain incidence properties (see e.g. [1] and [2]). In this paper we consider incidence properties of graphs of real functions and obtain the following sufficient condition for a bounded continuous real function to have a line cutting its graph in exactly two points.

THEOREM 1. Let $f: R \rightarrow R$ be a bounded continuous function; then there is a line cutting the graph of $f$ in exactly two points if there exist $\xi, \theta_{1}$ and $\theta_{2}$ with $\theta_{1}<\theta_{2}$ such that for all $\theta \in\left[\theta_{1}, \theta_{2}\right]$,

$$
\{x \mid f x-f \xi-(x-\xi) \tan \theta=0 \text { and } x \cos \theta>\xi \cos \theta\}
$$

is finite and nonempty.

In fact we prove a somewhat stronger result (Theorem 2) and also give examples to show that no two of the conditions are sufficient to imply its conclusion.

2. Theorem 2. Let $f: R \rightarrow R$ and define $g: R^{3} \rightarrow R$ by $g(\eta, \theta, x)=f x-f \eta$ $-(x-\eta) \tan \theta$. If

(i) $f$ is continuous;

(ii) $\varlimsup_{x \rightarrow \infty} f x<\infty$ and $\lim _{x \rightarrow-\infty} f x>-\infty$;

(iii) there exist $\xi, \theta_{1}$ and $\theta_{2}$ with $0<\theta_{1}<\theta_{2}<\pi / 2$ such that, for all $\theta \in\left[\theta_{1}, \theta_{2}\right],\{x \mid g(\xi, \theta, x)=0$ and $x>\xi\}$ is finite and nonempty, then there exists a line which meets the graph of $f$ in exactly two points.

Proof. We assume the result is false and obtain a contradiction. Let $\xi, \theta_{1}$ and $\theta_{2}$ satisfy (iii); without loss of generality we may suppose that $\xi=0$ and $f \xi=0$. We shall write $g(\theta, x)$ for $g(0, \theta, x)$. For $\theta \in\left[\theta_{1}, \theta_{2}\right]$ define $G \theta$ as the greatest real number $x$ such that $g(\theta, x)=0$; by (iii) $G$ is well defined and, since $f$ is continuous and $\overline{\lim }_{x \rightarrow \infty} f x<, \infty, G$ is strictly decreasing.

(I) $G$ is continuous on the left in $\left(\theta_{1}^{\prime}, \theta_{2}\right.$ ]; for suppose it is not continuous on

Received by the editors September 6, 1976.

AMS (MOS) subject classifications (1970). Primary 26A15.

Key words and phrases. Real functions, continuity, incidence. 
the left at $\psi^{\prime} \in\left(\theta_{1}, \theta_{2}\right]$ then there is an $\varepsilon>0$ and an increasing sequence $\left(\psi_{n}\right)$ with limit $\psi^{\prime}$ such that for all $n, G \psi_{n}-G \psi^{\prime}>\varepsilon$. Further $\lim G \psi_{n}$ exists and equals $\sigma$ say; clearly $\sigma \geqslant G \psi^{\prime}+\varepsilon$. We have, by definition of $G, f G \psi_{n}=$ $G \psi_{n} \tan \psi_{n}$, so, taking limits, fo $=\sigma \tan \psi^{\prime}$ since $f$ is continuous. Thus $G \psi^{\prime} \geqslant$ $\sigma \geqslant G \psi^{\prime}+\varepsilon$ and we have a contradiction.

(II) $G$ is continuous on some subinterval of $\left[\theta_{1}, \theta_{2}\right]$. Suppose it is not. We define strictly monotonic sequences $\left(\alpha_{n}\right),\left(\beta_{n}\right)$ with the following properties.

(1) $\theta_{1} \leqslant \alpha_{n}<\beta_{n} \leqslant \theta_{2}$,

(2) $\alpha_{n-1}<\alpha_{n}$ and $\beta_{n-1}>\beta_{n}$,

(3) for all $\phi \in\left(\alpha_{n}, \beta_{n}\right), g(\phi, x)=0$ has at least $2(n-1)$ solutions with $0<x<G \beta_{n}$.

Define $\alpha_{1}=\theta_{1}$ and $\beta_{1}=\theta_{2}$, then (1) and (3) are clearly satisfied. Suppose we have defined $\alpha_{1}, \alpha_{2}, \ldots, \alpha_{k}, \beta_{1}, \beta_{2}, \ldots, \beta_{k}$ such that (1), (2) and (3) are satisfied. Now $G$ is not continuous on $\left(\alpha_{k}, \beta_{k}\right)$ so let $\psi^{\prime} \in\left(\alpha_{k}, \beta_{k}\right)$ be a point of discontinuity. From (I), $G$ is discontinuous on the right at $\psi^{\prime}$. Thus there is an $\varepsilon>0$ and a decreasing sequence $\left(\psi_{n}\right)$ with limit $\psi^{\prime}$ such that for all $n$, $G \psi^{\prime}-G \psi_{n}>\varepsilon$. Now $\lim G \psi_{n}$ exists, and denoting it by $\sigma$, we clearly have $g\left(\psi^{\prime}, \sigma\right)=0$ and $\sigma \leqslant G \psi^{\prime}-\varepsilon$.

Now for all $x \in\left[\sigma, G \psi^{\prime}\right]$ we have $g\left(\psi^{\prime}, x\right) \leqslant 0$, for if there is a $w \in\left[\sigma, G \psi^{\prime}\right]$ with $g\left(\psi^{\prime}, w\right)>0$ then there will exist a member of the sequence $\left(\psi_{n}\right)$, say $\psi_{N}$, such that $g\left(\psi_{N}, w\right)>0$; since $g\left(\psi_{N}, x\right) \rightarrow-\infty$ as $x \rightarrow \infty$ and $g\left(\psi_{N}, x\right)$ is a continuous function of $x, G \psi_{N}>w \geqslant \sigma$ and we have a contradiction. Hence by (iii) there is a $\rho \in\left(\sigma, G \psi^{\prime}\right)$ such that $g\left(\psi^{\prime}, \rho\right)<0$. Thus we may choose $\alpha_{k+1}$ such that $\alpha_{k}<\alpha_{k+1}<\psi^{\prime}$ and $\tan \alpha_{k+1}>f \rho / \rho$; then for all $\phi \in$ $\left(\alpha_{k+1}, \psi^{\prime}\right), g(\phi, x)=0$ has at least two solutions with $\sigma<x<G \psi^{\prime}$ since $g(\phi, \sigma)>0, g(\phi, \rho)<0$ and $g\left(\phi, G \psi^{\prime}\right)>0$.

Putting $\beta_{k+1}=\psi^{\prime}$ we clearly have (1) and (2) satisfied with $n=k+1$. Now (3) is satisfied for $n=k$ and for large enough $n, \sigma \geqslant G \psi_{n}>G \beta_{k}$ so the above now gives that (3) is satisifed for $n=k+1$ and our definition of the sequences is complete.

Let $\phi=\lim _{n \rightarrow \infty} \alpha_{n}$. Then by (3) $\mid\{x \mid g(\phi, x)=0$ and $x>0\} \mid>2 n$ for all $n$ which contradicts condition (iii) of the theorem.

(III) Thus $G$ is continuous on some subinterval, say $\left[\phi_{1}, \phi_{2}\right]$, of $\left[\theta_{1}, \theta_{2}\right]$. Let $a=G \phi_{2}$ and $b=G \phi_{1}$; then $a<b$ and the domain of $G^{-1}$ contains $[a, b]$. We now define a decreasing function $h$ on a closed subinterval $\left[a^{\prime}, b^{\prime}\right]$ of $(a, b)$. For $\eta \in\left[a^{\prime}, b^{\prime}\right]$ and $\psi \in\left[G^{-1} \eta, \pi / 2\right)$ let

$$
S(\eta, \psi)=\{x \mid g(\eta, \psi, x) \leqslant 0 \text { and } x<\eta\} .
$$

Now $g\left(\eta, G^{-1} \eta, x\right)>0$ for $x \in[a, \eta)$ so $g(\eta, \psi, x)>0$ for $x \in[a, \eta)$; thus $x \in S(\eta, \psi)$ implies $x<a$ and $S(\eta, \psi)$ is closed. $S(\eta, \psi)$ is also bounded since $\lim _{x \rightarrow-\infty} f x>-\infty$; further $S\left(\eta, G^{-1} \eta\right)$ is nonempty since $0 \in$ $S\left(\eta, \overline{G^{-1}} \eta\right)$. Let

$$
\Psi \eta=\left\{\psi \mid S(\eta, \psi) \text { is nonempty and } G^{-1} \eta \leqslant \psi<\pi / 2\right\}
$$

and let $\psi_{0}(\psi)=\sup \Psi \eta$. Clearly 


$$
\psi_{0}(\eta)<\frac{\pi}{2} \quad \text { and } S\left(\eta, \psi_{0}(\eta)\right)=\bigcap_{\psi \in \Psi \eta} S(\eta, \psi)
$$

it is easy to see that $S\left(\eta, \psi_{0}(\eta)\right)$ is nonempty and also that $x \in S\left(\eta, \psi_{0}(\eta)\right)$ implies $g\left(\eta, \psi_{0}(\eta), x\right)=0$. Finally define $h$ by $h \eta=\max S\left(\eta, \psi_{0}(\eta)\right)$.

Let $\eta_{1}<\eta_{2}$ and $\psi^{\prime}=\tan ^{-1}\left(f \eta_{1}-f h \eta_{2}\right) /\left(\eta_{1}-h \eta_{2}\right)$; then $\psi_{0}\left(\eta_{2}\right)<\psi^{\prime} \leqslant$ $\psi_{0}\left(\eta_{1}\right)$. Thus for $x<h \eta_{2}, g\left(\eta_{1}, \psi^{\prime}, x\right)>0$ so $g\left(\eta_{1}, \psi_{0}\left(\eta_{1}\right), x\right)>0$. Hence $h \eta_{1}$ $\geqslant h \eta_{2}$ and $h$ is decreasing.

(IV) Thus $h$ has a point of continuity in $\left(a^{\prime}, b^{\prime}\right)$, say $c$. Consider the line $l$ given by $y-f c=(x-c) \tan \psi_{0}(c)$. Now $\{c, h c\} \subseteq \operatorname{pr}_{1}(l \cap f)$. It is easy to see that $G \phi$ is the only solution of $g(\phi, x)=0$ with $x>a$, so $x \notin \operatorname{pr}_{1}(l \cap f)$ for $x>c$ since $G$ is decreasing and $f$ is continuous. Since $\lim _{x \rightarrow-\infty} f x>$ $-\infty \operatorname{pr}_{1}(l \cap f)$ is compact so let $w=\min \operatorname{pr}_{1}(l \cap f)$. From our original hypothesis $\operatorname{pr}_{1}(l \cap f)$ has at least three members, so by the definition of $h c$ we must have $w<h c$. Choosing $\varepsilon>0$ such that $w<h c-\varepsilon<h c+\varepsilon<c$, there is, by the continuity of $h$ at $c$, a $\delta>0$ such that for all $x \in(c-\delta, c+$ $\delta)$ we have $|h x-h c|<\varepsilon$. Take $\eta=c+\delta / 2$ and $\psi^{\prime}=\tan ^{-1}(f \eta-f w) /(\eta-$ $w)$; then $g\left(\eta, \psi^{\prime}, x\right)>0$ for $x \in(w, c)$ and, hence, $g\left(\eta, \psi_{0}(\eta), x\right)>0$ for $x \in(w, c)$. Thus $|h \eta-h c|>\varepsilon$, which is a contradiction, and the theorem follows.

Symmetry shows that we can obtain three theorems similar to Theorem 2 by varying the conditions as follows.

(1) Replace $\{x \mid g(\xi, \theta, x)=0$ and $x>\xi\}$ in (iii) by $\{x \mid g(\xi, \theta, x)=0$ and $x<\xi\}$.

(2) Replace (ii) by

(ii) $\lim _{x \rightarrow \infty} f x>-\infty$ and $\varlimsup_{x \rightarrow-\infty} f x<\infty$, and (iii) by

(iii)' there exist $\xi, \theta_{1}$ and $\theta_{2}$ with $-\pi / 2<\theta_{1}<\theta_{2}<0$ such that, for all $\theta \in\left[\theta_{1}, \theta_{2}\right],\{x \mid g(\xi, \theta, x)=0$ and $x>\xi\}$ is finite and nonempty.

(3) Replace, as in (2), (ii) by (ii)' and (iii) by (iii)' except that $\{x \mid g(\xi, \theta, x)$ $=0$ and $x<\xi\}$ replaces $\{x \mid g(\xi, \theta, x)=0$ and $x>\xi\}$ in (iii)'.

It is clear that Theorem 1 now follows from these theorems.

3. We now give examples to show that if any of the conditions of Theorem 1 (or Theorem 2) are omitted then the conclusion is false.

EXAmple 1. Define $f: R \rightarrow R$ by

$$
f x= \begin{cases}x^{2} \sin x & \text { for } x \leqslant 0 \\ \sin x & \text { for } x \geqslant 0\end{cases}
$$

then, on choosing $\xi=0, \theta_{1}=\pi / 6, \theta_{2}=\pi / 5$, we see the only condition not satisfied in Theorem 2 is that $\lim _{x \rightarrow \infty} f x>-\infty$. However no line meets the graph of $f$ in exactly two points.

EXAmple 2. Let $S=R \times[-1,1]$ and $T$ be the set of all lines in the plane not of the form $x=$ constant or $y=k$ where $k$ is a constant with $|k|>1$. Let $\Omega$ be the smallest ordinal with cardinal $c$. Choose bijections $g: \Omega \rightarrow R$ and $s$ : $\Omega \rightarrow S$ and a surjection $t: \Omega \rightarrow T$ such that, for all $l \in T,\left|t^{-1} l\right|=3$. We now 
define functions $y: \Omega \rightarrow S$ and $z: \Omega \rightarrow S$ by transfinite induction. Let $\delta$ be the least ordinal such that $\operatorname{pr}_{1} s \delta=g 1$ and let $\delta^{\prime}$ be the least ordinal such that $\operatorname{pr}_{1} s \delta^{\prime} \neq g 1$. Define $y 1=s \delta$ and $z 1=s \delta^{\prime}$. Let $\lambda \in \Omega$ and suppose we have already defined $y \alpha$ and $z \alpha$ for all $\alpha<\lambda$. Let $Y_{\lambda}=\cup_{\alpha<\lambda} y \alpha, Z_{\lambda}=\cup_{\alpha<\lambda} z \alpha$ and $L_{\lambda}=\left\{l \in T|| l \cap\left(Y_{\lambda} \cup Z_{\lambda}\right) \mid \geqslant 3\right\}$.

If $g \lambda \in \operatorname{pr}_{1}\left(Y_{\lambda} \cup Z_{\lambda}\right)$ define $y \lambda=y 1$; if $g \lambda \notin \operatorname{pr}_{1}\left(Y_{\lambda} \cup Z_{\lambda}\right)$ define $y \lambda=s \eta$ where $\eta$ is the least ordinal such that $s \eta \in \operatorname{pr}_{1}^{-1} g \lambda \cap\left(S \backslash \cup_{l \in L_{\lambda}} l\right)$.

Let $L_{\lambda}^{\prime}=\left\{l \in T|| l \cap\left(Y_{\lambda} \cup Z_{\lambda} \cup\{y \lambda\}\right) \mid \geqslant 3\right\}$. If $t \lambda \in L_{\lambda}^{\prime}$ define $z \lambda=$ $z 1$; if $t \lambda \notin L_{\lambda}^{\prime}$ define $z \lambda=s \eta^{\prime}$ where $\eta^{\prime}$ is the least ordinal such that

$$
s \eta^{\prime} \in t \lambda \cap S \backslash \operatorname{pr}_{1}^{-1} \operatorname{pr}_{1}\left(Y_{\lambda} \cup Z_{\lambda} \cup\{y \lambda\}\right) \text {. }
$$

The definition of $y$ and $z$ is now complete.

Let $f=y \Omega \cup z \Omega$; then it is easy to verify that $f$ is a function such that each member of $T$ meets the graph of $f$ in exactly three points. Thus no line meets the graph of $f$ in exactly two points. Clearly $f$ is bounded and condition (iii) of Theorem 2 is satisfied.

An obvious example of a bounded continuous function such that no line meets the graph of $f$ in exactly two points is a constant function. However, if constant functions were the only bounded continuous functions with this property, there would be a much more natural theorem than Theorem 1 . We therefore give

EXAmple 3. Let $u_{n} x=\psi\left(16^{n} x\right) / 2^{n}$ for all $x \in R$ where $\psi: R \rightarrow R$ is periodic with period 1 and $\psi x=|x|$ for $|x| \leqslant \frac{1}{2}$. Define $f: R \rightarrow R$ by $f x=$ $\sum_{n=0}^{\infty} u_{n} x$; then $f$ is clearly continuous and bounded. Suppose there is a line $l$ which meets the graph of $f$ in exactly two points; we may clearly suppose the gradient $m$ of $l$ is greater than zero.

For $\eta \in R$ and $n \in N$ there exist consecutive minimum points $x_{n}$ and $x_{n}^{\prime}$ of $u_{n}$ such that $\eta \in\left[x_{n}, x_{n}^{\prime}\right]$. Let $y_{n}=x_{n}^{\prime}+16^{-n} / 2$. Now $u_{m} x_{n}=0=u_{m} x_{n}^{\prime}=$ $u_{m} y_{n}$ for $m>n$ so

$$
f y_{n}-f x_{n}=\sum_{i=0}^{n}\left(u_{i} y_{n}-u_{i} x_{n}\right) \geqslant \frac{1}{2} 2^{-n}-\sum_{i=0}^{n-1} \frac{3}{2^{i+1}} 16^{i-n} .
$$

Hence $\left(f y_{n}-f x_{n}\right) /\left(y_{n}-x_{n}\right) \geqslant(4 / 21) 8^{n} \rightarrow \infty$ as $n \rightarrow \infty$.

Letting $\Delta(y, x)=(f y-f x) /(y-x)$ for $x \neq y, \Delta(x, x)=0$, we have

$$
\Delta\left(y_{n}, x_{n}\right)=\Delta\left(y_{n}, \eta\right) \frac{y_{n}-\eta}{y_{n}-x_{n}}+\Delta\left(\eta, x_{n}\right) \frac{\eta-x_{n}}{y_{n}-x_{n}}
$$

so, from the above, there exist subsequences $\left(x_{n}^{\prime \prime}\right),\left(y_{n}^{\prime \prime}\right)$ with $x_{n}^{\prime \prime} \leqslant \eta<y_{n}^{\prime \prime}$ such that at least one of the following holds as $n \rightarrow \infty$ : (i) $\Delta\left(y_{n}^{\prime \prime}, \eta\right) \rightarrow \infty$ or (ii) $\Delta\left(\eta, x_{n}^{\prime \prime}\right) \rightarrow \infty$

Now $l$ meets $f$ in exactly two points, say $(\xi, f \xi)$ and $(\eta, f \eta)$ with $\xi<\eta$. Thus there exists a $y_{n}^{\prime \prime}>\eta$ such that $\Delta\left(y_{n}^{\prime \prime}, \eta\right)>m$ or there exists $x_{n}^{\prime \prime}$ with $\xi<x_{n}^{\prime \prime} \leqslant$ $\eta$ such that $\Delta\left(\eta, x_{n}^{\prime \prime}\right)>m$. Since $m>0$ and $f$ is bounded and continuous, $\Delta\left(y_{n}^{\prime \prime}, \eta\right)>m$ implies a point $(\sigma, f \sigma) \in l$ with $\sigma>\eta$ so we must have $\Delta\left(\eta, x_{n}^{\prime \prime}\right)$ $>m$. Similarly we must have a $z_{p}$ with $\xi<z_{p}<x_{n}^{\prime \prime}$ such that $\Delta\left(z_{p}, \xi\right)>m$. 
However $\Delta\left(\eta, x_{n}^{\prime \prime}\right)>m$ and $\Delta\left(z_{p}, \xi\right)>m$ implies a point $(\rho, f \rho) \in l$ with $\xi<\rho<\eta$ and we have a contradiction.

\section{REFERENCES}

1. F. Bagemihl, $A$ theorem on intersections of prescribed cardinality, Ann. of Math. (2) 55 (1952), 34.

2. W. Sierpiński, Une généralisation des théorèmes de S. Mazurkiewicz et F. Bagemihl, Fund. Math. 40 (1953), 1-2.

Department of Mathematics, The University, Southampton, England 